\title{
Treatment Burden and Quality of Life of Patients with Neovascular Age-Related Macular Degeneration (nAMD) and Their Caregivers-A Review
}

\author{
Rupak Kanti Biswas ${ }^{1}$, Rupak Roy ${ }^{2}$, Nitin Maksane ${ }^{3}$, Maulik Bhavsar ${ }^{3,}$, Ajitabha Sanyal ${ }^{3}$ \\ ${ }^{1}$ Department of Vitreo - Retina, B. B. Eye Foundation, Kolkata, West Bengal, India \\ ${ }^{2}$ Department of Vitreo - Retina, Sankara Nethralaya, Kolkata, West Bengal, India \\ ${ }^{3}$ Novartis Healthcare Pvt. Ltd., Mumbai, Maharashtra, India
}

Email address:

rupakkbiswas@gmail.com (R. K. Biswas),rayrupak@gmail.com (R. Roy), nitin.maksane@novartis.com (N. Maksane), maulik.bhavsar@novartis.com (M. Bhavsar), ajitabha.sanyal@novartis.com (A. Sanyal)

${ }^{*}$ Corresponding author

\section{To cite this article:}

Rupak Kanti Biswas, Rupak Roy, Nitin Maksane, Maulik Bhavsar, Ajitabha Sanyal. Treatment Burden and Quality of Life of Patients with Neovascular Age-Related Macular Degeneration (nAMD) and Their Caregivers-A Review. International Journal of Ophthalmology \& Visual Science. Vol. 6, No. 3, 2021, pp. 164-171. doi: 10.11648/j.ijovs.20210603.13

Received: June 21, 2021; Accepted: July 9, 2021; Published: August 5, 2021

\begin{abstract}
The growing incidence of neovascular age-related macular degeneration (nAMD) in India and its debilitating consequences, such as physical, psychological, and emotional stress, are a cause of significant concern. Failing eyesight due to nAMD hinders the ability of patients to perform daily tasks, leading to dependency on others - often resulting in anxiety and depression. Although treatment options such as anti-vascular endothelial growth factors (anti-VEGFs) are readily available, long-term treatment compliance is often compromised. Thus, it is important for health care providers to be aware of the burden associated with nAMD for both patients and caregivers. India, with its large urban and rural population base, faces varied challenges in health care accessibility and affordability. Further, there is a paucity of India-specific studies to ascertain patient and caregiver burden related to nAMD. A deeper understanding of disease awareness and treatment expectations from an Indian perspective may further help clinicians to provide optimum management to patients. The current review provides insights into the quality of life $(\mathrm{QoL})$ and treatment-related burden for patients with nAMD and their caregivers. Further, it emphasizes the need of PAN-India studies to ascertain the patient and caregiver burden related to nAMD, which may assist in devising treatment algorithms and pricing policies suited to the Indian population and enable patients to receive quality eye care.
\end{abstract}

Keywords: Wet AMD, nAMD, Caregiver, Treatment Burden, Quality of Life, Patient Perspective, Economic Burden, Indian Perspective

\section{Introduction}

Age-related macular degeneration (AMD) is currently the third common cause of blindness after cataract and glaucoma across the world. With a global blindness prevalence of $8.7 \%$, AMD has been aptly labeled a 'priority eye disease' and is a growing challenge in the developing world [1]. A 2014 study on the progression of AMD burden projected that 196 million people would be affected by AMD by 2020; the figure is expected to rise to 288 million by 2040 [2]. Earlier it was assumed that AMD primarily affects people of European descent. However, a study has reported that the prevalence of late AMD in Asians and Europeans between 40 and 79 years of age was similar (0.56\% and $0.59 \%$, respectively) [3]. Asians had fewer early signs of disease than Europeans $(6.8 \%$ vs. $8.8 \%$, respectively) [3]. As Asia accounts for over $60 \%$ of the world population, it may be reasonable to predict that about one-third of future AMD cases would come from the region $[2,4]$.

The 2004 Aravind Comprehensive Eye Study reported the overall prevalence of AMD to be $1.3 \%$ in adults aged $40-49$ years in South India. The prevalence of AMD increased progressively with age: $3.9 \%$ in $50-59$-year-old patients, $5 \%$ 
in 60-69-year-old patients, and $6.9 \%$ in 70 years or older patients [5]. The study further reported the prevalence of early AMD to be $2.7 \%$, and that of late AMD to be $0.6 \%$. The age-adjusted prevalence of AMD (adjusted to the population estimates for the year 2000) in India was 3.1\% [5]. Data from other epidemiological studies in India from 2004 to 2007 indicate that the prevalence of AMD ranged between $1.4 \%$ and $1.8 \%$ of the total population $[5,6]$. Another population-based cross-sectional study in 2016-'Sankara Nethralaya Rural-Urban AMD Study'-conducted in South India revealed that the prevalence of late AMD was $2.26 \%$ in rural areas and $2.32 \%$ in urban areas [7]. A 2018 observational study reported that the prevalence of AMD increased with age with the majority of patients belonging to the age group of $61-70$ years $(47.5 \%)$ [7]. The most important risk factor associated with AMD reported in this study was aging for both rural and urban populations. This is a major concern for countries such as India due to the increasing geriatric population [8].

It is well established that early AMD may progress to dry or wet AMD, and wet AMD is referred to as neovascular AMD (nAMD) [9]. The early stage of AMD is characterized by atrophy or hypertrophy of the retinal pigment epithelium (RPE) underlying the central macula, as well as drusen deposition. It has been estimated that $10 \%-20 \%$ of patients with early AMD progress to nAMD [10].

Disease progression is characterized by an abnormal growth of choroidal vessels or capillaries under the macula leading to fluid and blood leakage and the development of a subretinal scar. Finally, the RPE and the photoreceptors are destroyed, leading to blindness [10]. Loss of vision affects routine daily activities of the patient. Eventually, family members are often called in as caregivers to provide emotional and physical support to patients. The burden on the caregiver varies based on the stage of disease and treatment frequency [11]. Overall, the disease places a substantial burden on both patients and caregivers $[12,13]$. In this article, we aim to review the burden affecting patients with nAMD and their caregivers and discuss the scope of care that may be offered, with a specific focus on India.

\section{Methodology}

A comprehensive literature search for articles in English was performed on nAMD in MEDLINE database and Google Scholar with the following MESH terms: age-related macular degeneration/neovascular AMD, patient, caregiver AND burden/challenges. Additional search terms included: unmet need, diagnosis, nAMD in India, awareness, daily living, QoL (vision-related), psychological burden, depression, treatment/anti-VEGF related burden, cost, independence. Other search terms included: comprehensive eye care and primary eye care, caring for nAMD. The search was limited to the period from 2004 to 2020 . The articles were further screened to mine relevant information pertaining to global and India-specific nAMD. Titles and abstracts of the retrieved records from the search results were reviewed to include relevant publications within the scope of this study.

\section{Burden and Consequences of nAMD}

The main consequence of untreated nAMD is blindness [14]. Evidence shows anti-vascular endothelial growth factor (anti-VEGF) treatment discontinuation for more than 3 months leads to $91 \%$ of patients developing choroidal neovascular lesions and significantly declined visual acuity (VA) [15].

The impact of nAMD on daily activities and quality of life (QoL) of patients accounts for the physical burden of the condition. A systematic review assessing the impact of AMD (including wet AMD) concluded that the disease has a substantial influence on a wide range of daily activities. Additionally, the analysis of specific activities highlighted that activities requiring mobility (such as inability to travel or reduced traveling, poor balance, gait alterations, and lack of postural stability) were impacted in $22 \%$ of the studies and that patient-reported visual function activities (such as inability to detect traffic gaps to cross the road, inability to drive, correct identification of colors and food, etc.) were reported in $17 \%$ of the studies [16]. Evidence further showed that loss of vision affected the overall well-being of an individual, increased dependency levels, and caused social isolation. The evaluation of vision-related QoL among patients with nAMD may act as a tool for clinicians to aid in the therapeutic decision-making process [17].

Apart from the effects on a wide range of daily activities, fear of falling has been recognized as having a major impact on QoL in AMD patients with severe vision loss. It is perceived that AMD-related vision loss may lead to difficulty in balancing, which greatly increases the fear of falling, ultimately resulting in decreased walking, limited physical activity, and subsequently social isolation, depression, frailty, injury, and death [18]. The Beaver dam study explored the association of visual functioning with physical outcomes (such as falls and fractures among others) and limitations in the elderly population and found visual function impairment to be statistically associated with a fear of falling [18]. A study in AMD patients diagnosed with choroidal neovascularization (CNV) or geographic atrophy showed that patients with worse VA and worse contrast sensitivity had a significantly greater fear of falling. This evidence substantiates the direct correlation between loss of VA and fear of falling [19]. The challenges of physical disability lead to dependency on caregivers. Muller et al. reported that around $30.1 \%$ of patients were dependent on help for daily activities, 39.8\% needed assistance to deal with authorities, and $21.4 \%$ needed mental and moral support [20].

Depression along with physical disability creates anxiety and hopelessness among patients, deteriorating their health-related quality of life (HRQoL) [21, 22]. A global cross-sectional survey revealed a higher rate of depression among patients with nAMD (34\%) compared to patients without nAMD ( $8 \%$ to $20 \%$ ). Patients with nAMD also felt an increase in emotions such as fear and frustration, which 
increased with the disease duration [23]. These results are further supported by an Indian study, which suggested that around $26.4 \%$ of patients with AMD were diagnosed with depressive disorder. There were more patients with wet AMD and depression than patients with dry AMD. These patients with depression had higher general and vision-specific disabilities compared to non-depressed patients, indicating that psychological morbidity in these patients contributes significantly to their disability [21].

The physical and psychological burden of nAMD is compounded, as the disease mainly impacts older patients whose vision might be already deteriorating due to coexisting ocular and other systemic diseases [15]. Altogether, these act as significant stressors for patients, further increasing the disease burden [17, 24]. Although there is ample global evidence highlighting the physical and psychological burden of nAMD, there is limited evidence from the Indian subcontinent.

\section{Current Treatment Regimens and Their Impact}

Globally, anti-VEGF medications such as ranibizumab (RBZ) and aflibercept (AFB) are approved for the treatment of nAMD, with brolucizumab being a new addition to this list $[25,26]$. Many pivotal clinical trials have proven the effectiveness of anti-VEGFs not only in halting the progression of nAMD but also in improving vision and gain in vision-related QoL (VRQoL) [4, 27, 28]. A study on the effects of RBZ indicated that compared to no treatment, monthly RBZ therapy may potentially reduce the progression of nAMD to legal blindness by $72 \%$ [29].

The treatment of patients with nAMD with anti-VEGF medications had a substantial impact in reducing disease progression and preventing loss of vision. A clinical trial in Asian patients with nAMD assessing the QoL after receiving standard of care anti-VEGFs showed improvement in best-corrected VA (BCVA), which was associated with improved vision [30-32]. Treatments for nAMD appear to be effective in improving patients' overall VRQoL, although other factors may be involved in improving emotional scores and mobility [27]. Our literature search also highlighted that data on impact of anti-VEGF therapy on VRQoL are sparse from an Indian perspective.

The following section highlights the patient and caregiver burden associated with anti-VEGF therapy, their impact on overall QoL and on treatment adherence and persistence.

\section{5. nAMD Treatment-Related Burden: Patient Perspective}

Since the introduction of anti-VEGF agents, there has been a significant, positive impact on the incidence of blindness due to nAMD. The burden of nAMD such as frequent injections, frequent monitoring, traveling, etc. are presently more related to vision loss or poor quality of vision rather than blindness [11]. This burden also imposes patient-related challenges that can be broadly categorized as follows: physical, psychological, and economic.

\subsection{Physical and Psychological Burden Associated with nAMD Treatment}

The physical and psychological burden associated with nAMD include access to therapy, disease awareness, treatment beliefs, expectations and experiences, HRQoL, and travel and time considerations.

Disease awareness of AMD is highly variable among countries. An international survey commissioned by the AMD Alliance International in 14 countries in 2005 reported AMD awareness of only $4 \%-30 \%$, the highest being in the United States (US). A 2017 survey conducted by Zhang et al. among 385 Chinese citizens showed that $69.3 \%$ of participants were not at all familiar with the term AMD and that only $6.8 \%$ of participants were somewhat familiar with the disease [33]. Another study from the Indian subcontinent concluded that awareness of AMD was 7.6\% [34]. Data from an Indian study indicated that there is a need for knowledge awareness about common eye diseases among the Indian population [35]. However, Indian studies focusing on nAMD disease awareness are warranted. This is of particular importance to resource-limited settings such as India, as increasing the knowledge about eye diseases could lead to an increase in acceptance of routine eye examination for early detection and early treatment of conditions such as nAMD [34, 35].

Most developed countries have structured treatment policies, infrastructure, resources, and comprehensive reimbursement policies for management of diseases. Even though India is progressing in terms of health care provision, it still lacks the expert medical care that is needed for diseases such as nAMD. A cross-sectional survey was conducted in 11 Indian cities in 2016 on the existing health care infrastructure and practice patterns available to treat diabetic retinopathy. The results revealed that only $68.6 \%$ of facilities had a dedicated retina unit, with more than half $(51.6 \%)$ reporting the need for laser and surgical equipment for better treatment. Further, $46.5 \%$ of eye care centers did not have the resources to track patients for follow-up and monitoring [36]. The data obtained from this study can be extrapolated to nAMD, as patients with nAMD may be expected to face almost similar challenges with medical specialists and facilities.

In India, there is a substantial difference in health care facilities available in the urban and rural scenarios: metro and tier 1 cities have better access to eye health care for patients; however, providing the same for patients from rural India is an ongoing challenge. Efforts are being made by the Indian government to improve access to primary eye care under the National Program for Blindness Control. Vision care centers have been established in small towns and rural areas to achieve this objective [37]. But the huge disease burden, coupled with a predominantly self-pay market, compels patients to rely on the private sector [4]. 
Challenges in access to therapy in rural India can be addressed with telemedicine. Telecommunication technology will help in remote diagnosis and treatment of patients. The teleophthalmology unit of Aravind Eye Care in Andipatti, India, is one example of the use of telemedicine in eye care [38]. Although a novel attempt, there is a pressing need to intensify telemedicine service efforts in other parts of the country.

Expectations from treatment vary among patients. Most patients are apprehensive about IVI prior to its initiation. Studies have demonstrated that anticipated discomfort and anxiety during or before an IVI, fear of having an injection, fear of losing eyesight, fear of the unknown, and fear due to an unfamiliar treatment procedure are often experienced [39].

Expectations from IVI treatment could be classified into pre-therapy expectations and post-therapy expectations. Prior to therapy initiation, most patients $(70.2 \%)$ were generally optimistic about the outcome. A total of $47 \%$ of the patients anticipated a reduction in the number of injections or complete discontinuation of treatment after a few injections. Post-therapy, the expectations changed, with $53 \%$ of patients being as optimistic about the therapy as at the beginning, but $13.6 \%$ were less optimistic about the results. Optimistic patients who had good disease awareness and no fear of therapy showed a numerically better VA at baseline and also at the end of the study as compared to other patients [20]. Though these data summarize the global scenario, these effects may be extrapolated to the Indian population, considering that the beliefs, expectations, and experiences of patients may be largely similar.

Based on the aforementioned information, anticipating patient fears, beliefs, and expectations and increasing communication based on patient personality type may be critical in the adequate treatment of nAMD [20].

Health-related quality of life (HRQoL) is defined as " aspects of self-perceived well-being that are related to or affected by the presence of disease or treatment" [40]. In the context of this review, HRQoL reflects treatment on vision and the measurement of the impact it has on daily living and the emotions of a patient with nAMD.

Global evidence indicates that the physical component score - an index of overall physical functioning-was significantly lower among patients with nAMD compared to patients without nAMD even after anti-VEGF treatment. Patients undergoing treatment for nAMD reported greater overall activity impairment compared to their matched controls. In addition, anxiety scores and sleep problems were comparable between both groups and depression was only numerically higher in the nAMD group [28]. Another study concluded that treated patients with nAMD had a comparable HRQoL to their age-matched peers, but they perceived their health to deteriorate considerably with time. Patients who were currently on anti-VEGF therapy had better expectations of future HRQoL when compared to patients not on anti-VEGFs [41].

Intravitreal injections involve frequent clinic visits for monitoring and therapy. The frequency of visits, the associated travel time, and the time spent at the clinic add up as significant barriers to adherence and persistence to therapy $[39,42]$. A US study reported that patients with nAMD spend approximately 90 minutes per week on clinical visits. However, pre-appointment preparation, travel, waiting time, treatment time, and post-appointment recovery time added up to almost 12 hours per visit [43]. Another study reported a significantly higher drop-out rate over a 2-year period for patients who lived $\geq 100 \mathrm{~km}$ from the clinic than those who lived $<100 \mathrm{~km}$ from the clinic $(50 \%$ vs. $28 \%, \mathrm{p}=0.002)$, signifying that traveling time added a significant burden for patients. A similar study in France also concluded that 51.7\% of patients discontinued IVI treatment due to the long distance between home and hospital [44, 45]. These time considerations are quite relevant to the Indian setting, considering the mode of transportation, traffic situation, and increased population.

Management of other medical appointments due to comorbidities associated with aging is also considered a time-related burden. Based on this, it can be assumed that patients must manage and plan appointments for other medical treatments, which may take precedence over ophthalmology treatments [42].

Another aspect to time burden is loss of productivity. Spooner et al. reported that due to the time spent on travel and frequent clinic visits, the loss of productivity for the patient amounted to $4.4 \pm 1.7$ hours per visit. Some patients may also require a day of recovery after the procedure, adding to further loss of productivity [42]. From an Indian perspective, patients with AMD present later in the course of disease progression [46] and hence would face the challenge of independent traveling. In addition, eye care centers are majorly concentrated in larger cities and there is a paucity of specialized equipment and medical experts, which adds to the challenge of delayed diagnosis [47]. Consequently, patients from rural India need to travel far to access optimal treatment at specialty centers. Consequently, patients spend a significant amount of time accessing health care services.

\subsection{Economic Burden}

The economic burden includes direct and indirect costs associated with dosing and treatment adherence, loss of time, etc. The economic burden also extends to personal expenses incurred by the patient, such as those arising from the need for mobility, low-vision aids, and modifications within the home. Spooner et al. carried out a survey that reported that patients considered cost to be a significant burden [42].

There is a paucity of such studies in India. However, based on available data, the following speculations can be made. Considering the prevalence of nAMD and based on the population of India, around 0.1 million patients will need anti-VEGF treatment. It has been estimated that out-of-pocket health expenditure in India accounts for $69.1 \%$ of all health care expenditure $[48,49]$. Further there is a lack of insurance and reimbursement for treatment, posing a huge challenge for the management of a chronic disease such as nAMD [50] that requires long-term care to prevent progression. So, it can be 
inferred that frequency of dosing and cost are important attributes for clinicians while selecting an anti-VEGF agent. Due to the above-mentioned factors, anti-VEGFs treatment is associated with significant direct treatment costs and indirect costs such as traveling to specialty clinics. Furthermore, due to the chronic nature of the disease and the associated direct and indirect cost burden, treatment compliance is severely affected.

Collectively, all these factors increase the economic burden associated with the treatment. In resource-constrained settings such as India, these challenges are likely to impact treatment compliance, timely treatment by the clinician, and time spent on counseling and rehabilitation of a patient.

\section{6. nAMD Treatment-Related Burden: Caregiver Perspective}

Caring for patients with vision loss is a substantial burden. Patients with nAMD are usually cared for both in and outside the hospital by family members without any formal training $[12,51]$. The responsibilities of a caregiver include providing the patient with assistance in activities of daily living (e.g. reading, transportation, personal care and financial management), providing medical therapy compliance, assistance in using residual vision more effectively with the help of devices and emotional support [12]. Caregivers also provide support at medical appointments, and emotional and psychological support [51]. Evidence shows that patients with visual impairment had a $61.9 \%$ increased dependence on family members and friends over a period of 5-15 years [52].

Another study conducted to analyze the emotional and physical impact on caregivers concluded that many caregivers feel sad and useless, not to mention the time off from work for caregiving and loss of personal and leisure time due to increasing dependency levels [42].

\subsection{Time}

Patients with nAMD need assistance with daily tasks such as buying groceries, cooking, cleaning, finances, and mobility. They also need assistance for tasks that require fine vision details such as reading, recognizing faces, hygiene or grooming, and driving [51].

The caregiver must invest a significant amount of time to care for the patient, and additional domestic duties that encroach upon caregivers' time, leading to reduced personal time for caregivers [51]. Spooner et al. reported that the time spent by the caregiver was on an average $4.3 \pm 3.2$ hours per week on assisting patients in activities of daily living and $6.2 \pm 1.1$ hours per month to accompany them for IVI [42]. Another study on 150 caring relatives of patients with nAMD reported that the caring time ranged between a minimum of 0.25 hours and a maximum of 55 hours per week (average time $6.4 \pm 8.5$ hours per week). Females spent more time in caregiving than males $(6.86 \pm 9.37$ vs. $5.59 \pm 6.89$ hours per week, respectively). Caring time included time spent on household assistance, leisure activities, and office work; of note, caring hours were dependent on the VA of the patient. It was observed that caring hours per week increased with increased loss of VA. The study also reported that on an average, 14.5 hours a year was spent by a caregiver to accompany a patient for IVI therapy, resulting in an increased time-related burden and loss of productivity [13]. Although there is ample global evidence discussing the time spent by caregivers in other countries, no relevant published literature from India was found in our search. However, based on the social setting in India, the time spent by the caregiver would be substantial and an extrapolation of global data may be acceptable.

\subsection{Quality of Life}

The QoL of caregivers is also affected due to various factors. Since most patients with nAMD are elderly, they require assistance from a caregiver [51]. This not only impacts the QoL of patients but also that of caregivers as well, as in the majority of cases, the caregivers are family members [23]. A survey assessing the QoL of caregivers of patients with nAMD reported a mean QoL value of $6.73 \pm 1.90$ (on a scale of 0 to 10 , with $0=$ "totally unhappy" to $10=$ "perfectly happy"). The survey also reported that among the various factors that were considered such as vision, age, insurance of patients, gender, and age of caregivers, a significant decline in QoL $(p<0.001)$ was associated only with increased hours of care per week [13]. Another study in the United Kingdom (UK) revealed that if primary caregivers were of a younger age, they reported a greater burden, plausibly due to the fact that they were employed; of note, caregiving caused a significant disruption in their daily life [11].

\subsection{Emotional Burden}

Caregivers feel a constant concern about the care they provide to the patient. Some caregivers experience feelings of guilt about not being able to extend the level of care as they would like to due to time or economic constraints or physical inability. The safety of IVI emerges as a major reason for worry among caregivers. Anxiety about upcoming injections and the pain that the patient will undergo acts as a significant caregiver stressor [51]. Evidence suggests that caregivers who expend significant financial, emotional, and physical resources to take care of their loved ones suffering from nAMD, are prone to higher levels of depression [12].

A study conducted among family members caring for patients with vision loss showed that $35.4 \%$ of caregivers were at risk of developing depression. Younger patients and patients experiencing depression were associated with significantly higher caregiver depression scores [12]. A study conducted in India assessing depression among caregivers of legally blind patients reported that the greater the vision loss in patients, the higher the incidence of depression among caregivers. A total of $48 \%$ of caregivers of patients with no light perception were found to be depressed [53]. 


\subsection{Health Status}

A UK-based study demonstrated that caregivers' perception of patients' nAMD symptoms affected the former's health status. The dyadic health status, i.e. the combined health of patients and caregivers, showed a high level of shared health. Most caregivers felt burdened when the health of the patient was compromised. In the study, $39.6 \%$ of caregivers reported tiredness, $38.8 \%$ reported anxiety, and $29.6 \%$ reported depression as the most commonly experienced problems [11]. There exists an unmet need to assess the impact of caregiving on the health status of the caregiver in the Indian nAMD scenario.

\subsection{Economic Burden}

Accompanying the patient for IVI therapy is a significant economic burden for the caregiver, especially since it involves sacrificing time that they could otherwise spend on work. The economic burden for the caregiver is based on the loss of economic resources (including cost of treatment, traveling, and time), loss of wages for the day, and loss of productivity [42]. Other direct non-medical costs such as travel, cost for housekeepers, acquisition costs, and home modifications also add to the economic burden on the caregiver [13, 42]. As the disease progresses, indirect costs on the caregiver increase substantially. These include rehabilitation, counseling, device installation, and supplements [43].

Of note, nAMD-related vision loss in Australia is estimated to cost $\$ 5.15$ billion per year to the health care system [54]. Similarly, a Canadian study estimated the economic burden of productivity loss, home modification, and loss of well-being amounted to $\$ 4.4$ billion, \$305 million, and $\$ 11.7$ billion, respectively [55]. These data suggest that the financial impact of lower VA is not only limited to the patient and the caregiver but also affects the community at large [42].

There is a paucity of studies that address the economic impact of nAMD on caregivers in India. However, considering the cost of treatment and time to travel for appointments, it is likely that the economic burden exerted by nAMD in India would be high.

\section{Conclusion and Future Directions}

Globally, visual impairment due to nAMD in the older population is a significant public health problem. There in an unmet need to improve accessibility of anti-VEGFs to combat the increasing prevalence of nAMD. This review article identifies the patient- and caregiver-related burden associated with the disease. In developing countries such as India, a high disease burden, coupled with low disease awareness and delayed or lack of treatment, can have far-reaching consequences. A collaboration of government and private eye care institutions would facilitate specialized eye care to the Indian population, especially in rural areas. This collaboration would not only ease the burden off patients but also their immediate family and the community at large, indirectly improving the country's health and economy.

India has varied sets of challenges in terms of health care accessibility and affordability. There is dearth of India-specific evidence on the patient and caregiver burden related to nAMD. Hence, there is a need for PAN-India studies to understand the impact of nAMD. Overall, the information gained from such focused studies will provide regional insights to treating clinicians and will help in devising treatment algorithms and pricing policies suited to the Indian population.

\section{Acknowledgements}

Medical writing support was provided by IQVIA and was funded by Novartis Healthcare Pvt Ltd.

\section{References}

[1] World Health Organisation. Blindness and vision impairment prevention: Priority Eye Diseases. [cited 202019 October ]; Available from: http://tdr.who.int/blindness/causes/priority/en/.

[2] Wong, W. L., et al., Global prevalence of age-related macular degeneration and disease burden projection for 2020 and 2040 a systematic review and meta-analysis. The Lancet Global Health, 2014. 2 (2): p. e106-e116.

[3] Kawasaki, R., et al., The prevalence of age-related macular degeneration in Asians: a systematic review and meta-analysis. Ophthalmology, 2010. 117 (5): p. 921-7.

[4] Parikh, R., et al., A multinational comparison of anti-vascular endothelial growth factor use: The United States, the United Kingdom, and Asia-Pacific. Ophthalmology Retina, 2019. 3 (1): p. 16-26.

[5] Nirmalan, P. K., et al., Prevalence of vitreoretinal disorders in a rural population of southern India: The Aravind comprehensive eye study. Archives of ophthalmology, 2004. 122 (4): p. 581-586.

[6] Gupta, S. K., et al., Prevalence of early and late age-related macular degeneration in a rural population in northern India: the INDEYE feasibility study. Invest Ophthalmol Vis Sci, 2007. 48 (3): p. 1007-11.

[7] Jayashree MP, H. J., Arathi C, Brijesh A Patil and Niveditha RK, Prevalence of age Related Macular Degeneration in A Tertiary Care centre. Journal of Clinical Research and Ophthalmology, 2019.

[8] Raman, R., et al., The prevalence and risk factors for age-related macular degeneration in rural-urban India, Sankara Nethralaya Rural-Urban Age-related Macular degeneration study, Report No. 1. Eye, 2016. 30 (5): p. 688-697.

[9] Chakravarthy, U., et al., Progression from Early/Intermediate to Advanced Forms of Age-Related Macular Degeneration in a Large UK Cohort: Rates and Risk Factors. Ophthalmology Retina, 2020.4 (7): p. 662-672.

[10] Mathenge, W., Age-related macular degeneration. Community eye health, 2014. 27 (87): p. 49. 
[11] Gohil, R., et al., Caregiver burden in patients receiving ranibizumab therapy for neovascular age related macular degeneration. PloS one, 2015. 10 (6): p. e0129361.

[12] Bambara, J. K., et al., Family caregiver social problem-solving abilities and adjustment to caring for a relative with vision loss. Investigative ophthalmology \& visual science, 2009. 50 (4): p. 1585-1592.

[13] eyer-Wendl, H. and P. Walter, Financial burden and quality of life of informal caregivers of patients with wet age-related macular degeneration. Health Economics Review, 2016. 6 (1): p. 37.

[14] Lim, L. S., et al., Age-related macular degeneration. Lancet, 2012. 379 (9827): p. 1728-38

[15] Vaze, A., S. Fraser-Bell, and M. Gillies, Consequences of long-term discontinuation of vascular endothelial growth factor inhibitor therapy in the patients with neovascular age-related macular degeneration. Acta ophthalmologica, 2014. 92 (8): p. e697-e698.

[16] Taylor, D. J., et al., How does age-related macular degeneration affect real-world visual ability and quality of life? A systematic review. BMJ open, 2016. 6 (12): p. e011504.

[17] Picoto, M., et al., Health and vision related quality of life among patients with choroidal neovascular age related macular degeneration. Revista Brasileira de Oftalmologia, 2015. 74 (4): p. 216-221.

[18] Klein, B. E., et al., Associations of visual function with physical outcomes and limitations 5 years later in an older population: the Beaver Dam eye study. Ophthalmology, 2003. 110 (4): p. 644-50.

[19] Van Landingham, S. W., et al., Fear of falling in age-related macular degeneration. BMC ophthalmology, 2014. 14 (1): p. 10.

[20] Müller, S., et al., Treatment of age-related neovascular macular degeneration: the patient's perspective. Graefe's Archive for Clinical and Experimental Ophthalmology, 2017. 255 (11): p. 2237-2246.

[21] Banerjee, A., et al., Prevalence of depression and its effect on disability in patients with age-related macular degeneration. Indian journal of ophthalmology, 2008. 56 (6): p. 469.

[22] Cimarolli, V. R., et al., Anxiety and depression in patients with advanced macular degeneration: current perspectives. Clinical Ophthalmology (Auckland, NZ), 2016. 10: p. 55.

[23] Varano, M., et al., The emotional and physical impact of wet age-related macular degeneration: findings from the wAMD Patient and Caregiver Survey. Clinical Ophthalmology (Auckland, NZ), 2016. 10: p. 257.

[24] Azad, R., P. Chandra, and R. Gupta, The economic implications of the use of anti-vascular endothelial growth factor drugs in age-related macular degeneration. Indian journal of ophthalmology, 2007. 55 (6): p. 441.

[25] Kiss, S., et al., Real-World Injection Frequency and Cost of Ranibizumab and Aflibercept for the Treatment of Neovascular Age-Related Macular Degeneration and Diabetic Macular Edema. J Manag Care Spec Pharm, 2020. 26 (3): p. 253-266.
[26] Nguyen, Q. D., et al., Brolucizumab: Evolution through Preclinical and Clinical Studies and the Implications for the Management of Neovascular Age-Related Macular Degeneration. Ophthalmology, 2020. 127 (7): p. 963-976.

[27] Fenwick, E. K., et al., Change in vision-related quality of life and influencing factors in Asians receiving treatment for neovascular age-related macular degeneration. British Journal of Ophthalmology, 2018. 102 (3): p. 377-382.

[28] Jaffe, D. H., et al., The economic and humanistic burden of patients in receipt of current available therapies for nAMD. Journal of Comparative Effectiveness Research, 2018. 7 (11): p. 1125-1132.

[29] Bressler, N. M., et al., Estimated cases of legal blindness and visual impairment avoided using ranibizumab for choroidal neovascularization: non-Hispanic white population in the United States with age-related macular degeneration. Archives of ophthalmology, 2011. 129 (6): p. 709-717.

[30] Eleftheriadou, M., et al., Long-term outcomes of aflibercept treatment for neovascular age-related macular degeneration in a clinical setting. American journal of ophthalmology, 2017. 174: p. $160-168$.

[31] Talks, J. S., et al., First-year visual acuity outcomes of providing aflibercept according to the VIEW study protocol for age-related macular degeneration. Ophthalmology, 2016. 123 (2): p. 337-343.

[32] Gillies, M. C., et al., Twelve-month outcomes of ranibizumab vs. aflibercept for neovascular age-related macular degeneration: data from an observational study. Ophthalmology, 2016. 123 (12): p. 2545-2553.

[33] Zhang, C.-X., et al., Awareness of age-related macular degeneration and its risk factors among beijing residents in China. Chinese medical journal, 2017. 130 (2): p. 155.

[34] Thapa, R., et al., Population awareness of diabetic eye disease and age related macular degeneration in Nepal: the Bhaktapur Retina Study. BMC Ophthalmology, 2015. 15 (1): p. 188.

[35] Dandona, R., et al., Awareness of eye diseases in an urban population in southern India. Bull World Health Organ, 2001. 79 (2): p. 96-102.

[36] Gilbert, C. E., et al., Eye care infrastructure and human resources for managing diabetic retinopathy in India: The India 11-city 9-state study. Indian journal of endocrinology and metabolism, 2016. 20 (Suppl 1): p. S3.

[37] Misra, V., et al., Models for primary eye care services in India Indian journal of community medicine: official publication of Indian Association of Preventive \& Social Medicine, 2015. 40 (2): p. 79

[38] Chellaiyan, V. G., A. Nirupama, and N. Taneja, Telemedicine in India: Where do we stand? Journal of family medicine and primary care, 2019.8 (6): p. 1872.

[39] Monés, J., et al., Undertreatment of Neovascular Age-Related Macular Degeneration after 10 Years of Anti-Vascular Endothelial Growth Factor Therapy in the Real World: The Need for A Change of Mindset. Ophthalmologica, 2020. 243 (1): p. 1-8.

[40] Karimi, M. and J. Brazier, Health, health-related quality of life, and quality of life: what is the difference? Pharmacoeconomics, 2016. 34 (7): p. 645-649. 
[41] Péntek, M., et al., Subjective health expectations of patients with age-related macular degeneration treated with antiVEGF drugs. BMC geriatrics, 2017. 17 (1): p. 233.

[42] Spooner, K. L., et al., The burden of neovascular age-related macular degeneration: a patient's perspective. Clinical Ophthalmology (Auckland, NZ), 2018. 12: p. 2483.

[43] Prenner, J. L., et al., Disease burden in the treatment of age-related macular degeneration: findings from a time-and-motion study. American journal of ophthalmology, 2015. 160 (4): p. 725-731. e1.

[44] Boulanger-Scemama, E., et al., Ranibizumab for exudative age-related macular degeneration: a five year study of adherence to follow-up in a real-life setting. Journal francais d'ophtalmologie, 2015. 38 (7): p. 620-627.

[45] McGrath, L. A. and L. R. Lee, Characteristics of patients who drop out from ranibizumab therapy. The Asia-Pacific Journal of Ophthalmology, 2013. 2 (5): p. 295-299.

[46] Sudhalkar, A., et al., Retrospective hospital-based analysis of age-related macular degeneration patterns in India: 5-year follow-up. Indian journal of ophthalmology, 2015. 63 (12): p. 899-904.

[47] Gilbert, C. E., et al., Eye care infrastructure and human resources for managing diabetic retinopathy in India: The India 11-city 9-state study. Indian J Endocrinol Metab, 2016. 20 (Suppl 1): p. S3-s10.

[48] Gupta IaB, M. International Health Care System Profiles: India. [cited 2020 30th September ]; Available from: https://www.commonwealthfund.org/international-health-polic $\mathrm{y}$-center/countries/india.

[49] Roy, V., U. Gupta, and A. K. Agarwal, Cost of medicines \& their affordability in private pharmacies in Delhi (India). Indian J Med Res, 2012. 136 (5): p. 827-35.

[50] VRSI Market Research Final Report; Available from: https://vrsi.in/wp-content/uploads/2017/07/VRSI-Market-Rese arch.pdf; Accessed on: 19 October 2020. 2017 [cited 202019 October]; Available from: https://vrsi.in/wp-content/uploads/2017/07/VRSI-Market-Rese arch.pdf.

[51] Vukicevic, M., et al., Caregiver perceptions about the impact of caring for patients with wet age-related macular degeneration. Eye, 2016. 30 (3): p. 413-421.

[52] Hong, T., et al., Visual impairment and subsequent use of support services among older people: longitudinal findings from the Blue Mountains Eye Study. American Journal of Ophthalmology, 2013. 156 (2): p. 393-399. e1.

[53] Braich, P. S., et al., Burden and depression in the caregivers of blind patients in India. Ophthalmology, 2012. 119 (2): p. 221-226.

[54] Economics, D. A. and P. Mitchell, Eyes on the future: A clear outlook on Age-related Macular Degeneration. 2011.

[55] Gordon, K. D., et al., The cost of vision loss in Canada. 1. Methodology. Canadian journal of ophthalmology, 2011. 46 (4): p. 310-314. 